\title{
MedienPädagogik
}

Zeitschrift für Theorie und Praxis der Medienbildung

\section{Der mediale Habitus als System von Grenzen medienpädagogischen Handelns von Lehrenden in der Erwachsenenbildung}

Ricarda Bolten

\begin{abstract}
Zusammenfassung
Lehrenden in der Erwachsenenbildung kommt die Aufgabe zu, Medien in ihrer Arbeit didaktisch sinnvoll einzusetzen und die Medienkompetenzentwicklung bei ihren Teilnehmenden anzustossen. Da die Gruppe der Lehrenden eine sehr heterogene ist, stellt sich die Frage, von welchen Faktoren deren individuelle, medienpädagogische Professionalisierung abhängt. Von daher wird das Konzept des medialen Habitus als mögliches Erklärungsmuster für die Professionalisierungsentscheidungen der Lehrenden beleuchtet. Der mediale Habitus als System von Grenzen für Medienhandeln limitiert auch die Mediennutzung im beruflichen Kontext und die Anreize sich in diesen Bereichen zu professionalisieren. Wenn strukturelle Professionalisierungsanreize (wie die Verankerung medienpädagogischer Inhalte in pädagogischen Studiengängen) als Orientierung wegfallen, nehmen habituelle Grenzen für die Gestaltung der medienpädagogischen Professionalisierung einen besonderen Stellenwert ein.
\end{abstract}

Media habitus as a system of boundaries for media pedagogical actions of trainers in adult education

\begin{abstract}
Trainers in adult education have to use media in a didactically meaningful way and to initiate the development of media competence among their participants. Since the group of teachers is very heterogeneous, the question arises on which factors their individual, media-educational professionalisation depends. Therefore, the concept of the media habitus is examined as a possible explanation pattern for the professionalisation decisions of the teachers. The media habitus as a system of boundaries for media action also limits media use in the professional context and the incentives to professionalise oneself in these areas. If structural incentives for professionalisation (such as the anchoring of media pedagogical content in pedagogical courses at university) are dropped as an orientation, habitual boundaries for the design of media pedagogical professionalisation take on a special significance.
\end{abstract}




\section{Relevanz digitaler Medien in der Erwachsenenbildung}

Der Einsatz von Medien in der Erwachsenenbildung hat eine lange Tradition. Beispielhaft seien hier die Lesegesellschaften im 18. Jahrhundert, die Informationen einer breiteren Masse in der Gesellschaft zutrugen (vgl. Faulstich 2009), oder die Arbeiterradio- und Kinoreformbewegung (vgl. Podehl 1984) genannt. Seit den 1980er Jahren spielt auch der Einsatz von «neuen` hin zu digitalen Medien eine Rolle. Damals wurden diese Medien vor allem in Zusammenhang mit selbstgesteuertem Lernen diskutiert (vgl. Otto 1979). Schon 1996 schrieb Stang:

«Wer heute die Debatten über Qualität, Professionalität und Organisationsentwicklung ohne Bezug auf die Entwicklung im Bereich der Informations- und Kommunikationstechnik führt, verspielt die Chancen, die derzeit für eine Neuorientierung der Erwachsenenbildung geboten sind.» (Stang 1996, 54)

Die hier angesprochene Neuorientierung bezieht sich auf alle Ebenen der Erwachsenenbildung und die Bedeutung nahm im Prozess der Digitalisierung weiter zu. Auf institutioneller Ebene findet eine Umstrukturierung auf virtuelle Prozesse statt, die Möglichkeiten und Anforderungen an Vernetzung nehmen zu und es werden andere Ansprüche an die Infrastruktur und Ausstattung von Bildungsinstitutionen gestellt. Dies hat auch Auswirkungen auf die Organisationsebene, die ihre Kommunikationswege den Entwicklungen ihrer Zielgruppe entsprechend anpassen. Zum Beispiel müssen neben analogen auch digitale Marketingstrategien entwickeln werden. Auf Angebotsebene sind digitale Medien zum einen ein wichtiger Inhalt geworden, zum anderen bieten sie eine Vielzahl von didaktischen Möglichkeiten, wie die Gestaltung von Blended Learning Angeboten, Online-Lehre oder der digitalen Begleitung von Lernprozessen als Online-Lernberatung.

Auf der Lehr-/Lernebene haben die Entwicklungen Auswirkungen auf den Einsatz digitaler Medien bei der didaktischen Umsetzung. Für die Lehrenden bedeutet dies, dass sie professionell digitale und analoge Medien zur Unterstützung des Lehr-/Lernprozesses einsetzen können müssen. Lehrenden in der Erwachsenenbildung kommt neben der Vermittlung auch die Aufgabe zu, dass sie Medienkompetenzentwicklung bei den Teilnehmenden fördern sollen, damit diese zum einen arbeitsmarktfähig bleiben, aber auch damit diese in einer durch digitale Medien geprägten Welt auf allen Ebenen teilhaben können (vgl. Gapski, Oberle, und Staufer 2018). Die Lehrenden müssen in der Lage sein, eine kritische Stellung zur individuellen und gesellschaftlichen Bedeutung von Medien einzunehmen, um ihren beruflichen Medieneinsatz zur Unterstützung der Lernprozesse der Teilnehmenden auf (fach-)inhaltlicher Ebene zu unterstützen und auch um die Lebens- und Arbeitswelt der Teilnehmenden zu verstehen. So setzt beispielsweise die Europäische Kommission das Thema Medienkompetenzförderung als eine der zentralen Aufgaben der Erwachsenenbildung (vgl. European Commission 2015). 
Das im Projekt MEKWEP ${ }^{1}$ entwickelte medienpädagogische Kompetenzmodell beschreibt die Breite der Anforderungen an die Lehrenden. Die Anforderungen orientieren sich an den allgemeinen Anforderungsebenen Feldkompetenz, Werthaltungen und Selbststeuerung, Fachkompetenz, didaktische Kompetenz sowie fachdidaktische Kompetenz. Die Kompetenzfacetten müssen medienbezogen umgesetzt werden können um medienpädagogisch kompetent zu handeln. Dies bedeutet, dass die Lehrenden ein breites medienbezogenes Wissen über das Feld in dem sie tätig sind mitbringen müssen. Dies umfasst neben dem Wissen über die Medienkompetenzvoraussetzungen und Mediennutzungsgewohnheiten der Teilnehmenden auch das medienbezogene Wissen über die Organisation, in der sie tätig sind, da im heterogenen Feld der Erwachsenen- und Weiterbildung auch sehr unterschiedliche Organisationskulturen im Umgang mit Medien vorzufinden sind. Die medienbezogene Fachkompetenz umschreibt das Wissen und Können, das sich direkt am Lehr-/Lerninhalt ausrichtet. Je nach Fach, das der oder die Lehrende unterrichtet, werden andere Facetten von Medienwissen wichtig (z.B. müssen Lehrende, die eine Weiterbildung im IT-Bereich anbieten ein sehr viel breiteres und tieferes Verständnis von technologischen Entwicklungen mitbringen, als ein Lehrender oder eine Lehrende, der oder die einen Sprachkurs mit digitalen Medien didaktisch unterstützen möchte). Der Bereich der mediendidaktischen Kompetenz umschreibt ein über den Fachbezug hinausgehendes Medienhandeln, was die aktuellen Entwicklungen von Lehr-/Lernmedien angeht (z.B. Lernplattformen oder OER) und die Einsatzmöglichkeiten, die einen Mehrwert auf Seite der Lernenden und Lehrenden bieten können. Hierbei sollte der didaktische Medieneinsatz immer am Inhalt ausgerichtet sein. Im Bereich der medienbezogenen Einstellungen und Selbststeuerung sind selbstreflexive Fähigkeiten der Lehrenden über ihr eigenes Medienhandeln beschrieben und auch ein allgemeines Verständnis über die Auswirkungen der digitalen Entwicklungen auf die Lebens- und Arbeitswelt. Grundlegend hierfür ist eine allgemeine Medienkompetenz, die alle Menschen in Zeiten der Digitalisierung aufweisen sollten (vgl. Schmidt-Hertha et al. 2017).

In der Kompetenzfacette «Medienbezogene Einstellungen und Werthaltungen» zeigt sich, dass die medienbezogenen individuellen Präferenzen der Lehrenden neben äusseren Setzungen eine nicht zu vernachlässigende Komponente medienpädagogischer Kompetenz darstellt.

1 MEKWEP - Medienpädagogische Kompetenz des beruflichen Weiterbildungspersonals zur Unterstützung des Einsatzes digitaler Medien in formalen, non-formalen und informellen Lernsettings: https://die-bonn. de/mekwep/. 


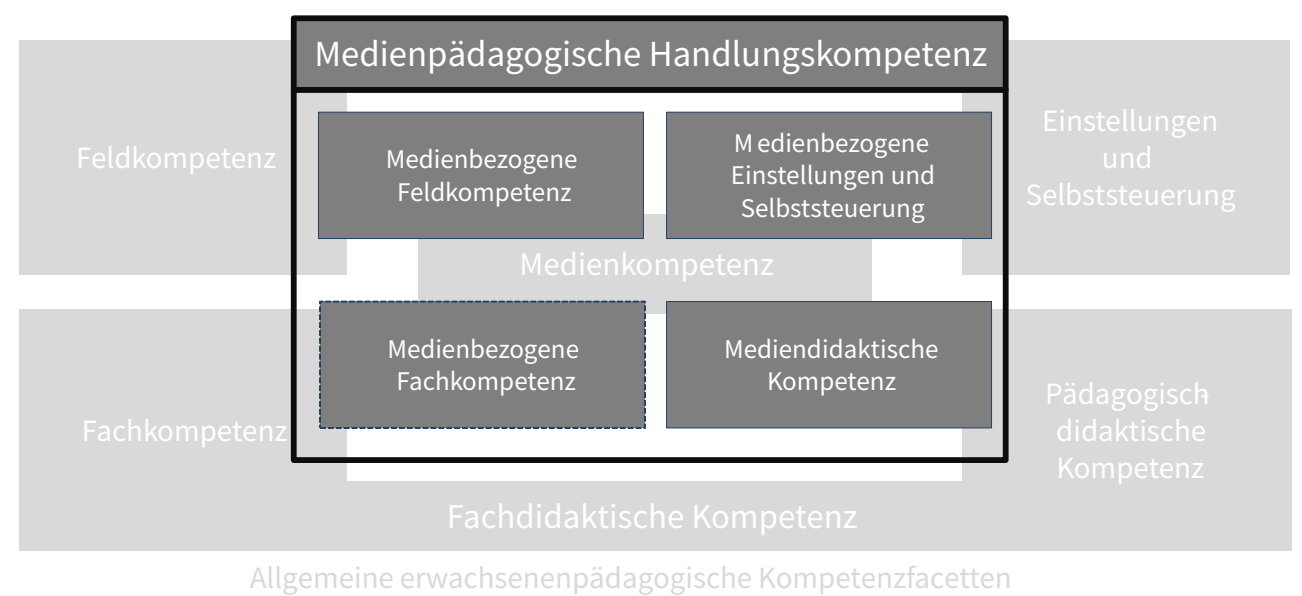

Abb. 1.: Medienpädagogische Kompetenzanforderungen an Lehrende in der Erwachsenenbildung (Quelle: Schmidt-Hertha et. al. 2017, 36).

Ausgehend von den medienpädagogischen Anforderungen an Lehrende in der Erwachsenenbildung stellt sich die Frage, wie sich die Lehrenden medienpädagogisch professionalisieren und welche Grenzen dieser Professionalisierung entgegenstehen. Hierfür wird im Folgenden kurz beleuchtet, welche biographischen Aspekte für medienpädagogische Professionalisierungsentscheidungen eine Rolle spielen. Anschliessend wird das Konzept des medialen Habitus vorgestellt um dieses als System von Grenzen für den Bereich der medienpädagogischen Professionalisierung für Lehrende in der Erwachsenenbildung zu beleuchten.

\section{Biographische Annäherung an medienbezogene Professionalisierung}

Betrachtet man die Ergebnisse des wb-Personalmonitors, kann davon ausgegangen werden, dass die lehrend in der Erwachsenenbildung Tätigen zum grossen Teil akademisch qualifiziert sind $(66,6 \%$ geben einen Fachhochschul- $(21,1 \%)$ oder Universitätsabschluss (38,3\%; 7,2\% Promotion) als höchsten Abschluss an) (vgl. Martin et al. 2017). Allerdings fallen nur 26,3\% der akademischen Abschlüsse in den Bereich der Pädagogik (Erziehungswissenschaft(en), Lehramt, Wirtschaftspädagogik) (vgl. ebd.): «Zusammenfassend kann festgehalten werden, dass die Erwachsenen- und Weiterbildung ein Bildungsbereich mit einem insgesamt eher niedrigen Professionalisierungsgrad des lehrend tätigen Personals ist, legt man dafür das Vorhandensein pädagogisch-akademischer Berufsabschlüsse zugrunde. Dies ist maßgeblich der spezifischen Personalstruktur in der Weiterbildung geschuldet, d.h. dass ein erheblicher Teil der Lehrveranstaltungen von nebenberuflich Tätigen bzw. auf Honorarbasis Beschäftigten geleistet wird, die allem Anschein nach in erster Linie aufgrund ihrer fachlichen Kompetenzen und weniger aufgrund der pädagogischen Ausbildung engagiert werden.» (ebd., 117) 
Ausgehend von diesen Ergebnissen, dass die Lehrenden primär nicht strukturell pädagogisch professionalisiert sind, kann davon ausgegangen werden, dass die individuelle Professionalisierung, bei der der Professionalisierungsweg primär selbst gestaltet wird, eine grosse Rolle spielt. Betrachtet man die Weiterbildungsbereitschaft der lehrenden Erwachsenenbildner und Erwachsenenbildnerinnen (28,4\%), ist diese im Vergleich zu allen Erwerbstätigen (20,7\%) höher (vgl. ebd.).

In einer qualitativen Interviewstudie mit Lehrenden, die (digitale) Medien zielgerichtet einsetzen, wurde der Frage nachgegangen, welche Zugänge diese zu Medien im Verlauf ihrer Biographie hatten (vgl. Rohs und Bolten 2017). Die Ergebnisse lassen vermuten, dass eine frühe Auseinandersetzung - meist im Kontext der Primärfamilie - einen grossen Einfluss auf die medienpädagogische Auseinandersetzung mit digitalen Medien haben kann. Die persönlichen Einstellungen und Werthaltungen, die sich in diesen biographisch frühen Phasen gebildet haben, scheinen eine entscheidende Rolle bei der Bewertung von digitalen Medien im Prozess der Auseinandersetzung mit medienpädagogischen Inhalten zu spielen (vgl. ebd.). Da für die Lehrenden ein Mangel an Orientierungspunkten besteht, in welchen Bereichen sie sich medienpädagogisch professionalisieren sollten um den Anforderungen in einer wandelnden Bildungslandschaft gerecht zu werden (Bolten und Rott 2018), kann davon ausgegangen werden, dass diese Professionalisierung besonders stark von individuellen Präferenzen geprägt ist. Weder bietet die strukturelle Professionalisierung klare Beschreibungen durch einheitliche medienpädagogische Curricula im Bereich (erwachsenen-)pädagogischer Studiengängen, noch finden sich medienpädagogische Facetten als zentrale Aspekte in Kompetenzbeschreibungen für Erwachsenenbildner und Erwachsenenbildnerinnen (vgl. Rohs, Bolten, und Kohl 2017). Von daher ist der Gestaltung der medienpädagogischen, individuellen Professionalisierung ein stark subjektiv geprägter Spielraum geboten. Dies erfordert eine erweiterte Perspektive auf die medienpädagogischen Professionalisierungswege, die neben der strukturellen Ebene auch die individuellen Präferenzen, Werthaltungen und Motivation der Lehrenden selbst in den Blick nimmt.

\section{Medialer Habitus}

Wie oben beschrieben ist die frühe biographische Auseinandersetzung mit digitalen Medien ein wichtiger Faktor für die medienpädagogische Professionalisierung von Lehrenden. Dies lässt einen Zusammenhang von medialem Habitus, der sich ebenfalls in der biografischen Auseinandersetzung bildet, und den medienpädagogischen Professionalisierungsentscheidungen der Lehrenden vermuten. Der mediale Habitus bietet einen theoretischen Bezugsrahmen für die Analyse medienpädagogischer Fragestellungen, indem er das individuelle Medienhandeln einer Person mit ihrer 
individuellen Lebenssituation und Sozialisationserfahrungen in Verbindung setzt (vgl. Biermann 2009).

Das Konzept des medialen Habitus (vgl. Kommer 2010) beruht auf dem Habitusverständnis nach Pierre Bourdieu. Der Habitus ist nach Bourdieu eine Verbindung von Handlungs- und Strukturdimension im sozialen Raum:

«[...] der Habitus ist Erzeugungsprinzip objektiv klassifizierbarer Formen von Praxis und Klassifikationssystem (principium divisionis) dieser Formen. In der Beziehung dieser beiden den Habitus definierenden Leistungen: der Hervorbringung klassifizierbarer Praxisformen und Werke zum einen, der Unterscheidung und Bewertung der Formen und Produkte (Geschmack) zum anderen, konstitutioniert sich die repräsentierte soziale Welt, mit anderen Worten der Raum der Lebensstile.» (Bourdieu 1987, 277f.; Hervorhebung v. Verf.)

Der Habitus, der auch als Ordnungssinn dient, bietet jedem Einzelnen ein Beurteilungsraster, das sich im Geschmack äussert und als Klassifikationsprinzip die (soziale) Welt deutet. Bezogen auf mediale Praxen leiten diese habituellen Dispositionsschemata inkorporiert medienbezogene Denk-, Wahrnehmungs- und Handlungsmuster an, die sich im medienbezogenen Lebensstil äussern (vgl. Michel 2010). Der Geschmack wird im Laufe der Sozialisation erworben und ist nicht von vornherein gegeben. Bourdieu bezieht sich in seinem Werk «Die feinen Unterschiede» auf den Geschmack bezüglich damals aktueller Medien wie Fotografien oder Musik und beschreibt in Orientierung an diesen die Unterscheidung des legitimen, mittleren und populären Geschmacks (vgl. ebd.). Die Aneignungsprozesse des Geschmacks und des Habitus bleiben auch heute stark durch die Sozialisation und das Elternhaus geprägt, auch wenn sich die Medienpalette in den letzten Jahrzehnten sehr ausgedehnt hat (vgl. Biermann 2013).

Der Habitus ist ein System von dauerhaften Dispositionen, das aber nicht unveränderbar ist:

«Der Habitus ist nicht das Schicksal, als das er manchmal hingestellt wurde. Als ein Produkt der Geschichte ist er ein offenes Dispositionssystem, das ständig mit neuen Erfahrungen konfrontiert und damit unentwegt von ihnen beeinflußt [sic!] wird. Er ist dauerhaft, aber nicht unveränderlich.» (Bourdieu und Wacquant 2006, 167f.)

Dies ist besonders von Bedeutung, wenn man den Habitus im pädagogischen Zusammenhang betrachtet, da er eben kein unveränderbares System darstellt, sondern unter bestimmten Voraussetzungen durchaus transformierbar ist (vgl. Koller 2012; von Rosenberg 2011). Die Mehrdimensionalität des Habitus bietet die Möglichkeit, diesen zu transformieren (vgl. von Rosenberg 2011). Durch Distanzierung des eigenen Habitus, wenn dieser durch Neues irritiert wird, und der Suche nach neuen Anschlussmöglichkeiten können Transformationsprozesse des Habitus' angestossen werden (vgl. ebd.). 
Nach Kommer und Biermann $(2012,90)$ ist der mediale Habitus der Teil des Gesamthabitus, der sich auf medienbezogene Handlungspraxen bezieht:

«Unter medialem Habitus verstehen wir ein System von dauerhaften medienbezogenen Dispositionen, die als Erzeugungs- und Ordnungsgrundlagen für mediale Praktiken und auf Medien und den Medienumgang bezogene Vorstellungen und Zuschreibungen fungieren und die im Verlauf der von der Verortung im sozialen Raum und der strukturellen Koppelung an die mediale und soziale Umwelt geprägten Ontogenese erworben werden. Der mediale Habitus bezeichnet damit auch eine charakteristische Konfiguration inkorporierter, strukturierter und zugleich strukturierender Klassifikationsschemata, die für ihre Träger in der Regel nicht reflexiv werden.»

Den Rückgriff auf das Konzept des (medialen) Habitus als theoriegeleitetes Analysekonzept begründet Kommer (2010) mit einem Mangel an Erklärungsmustern für medienbezogene Dispositionen, die sich auf die Vermittlung und den Erwerb von Medienkompetenz im schulischen Kontext auswirken.

Der mediale Habitus dient also als Ordnungssinn für medienbezogenes Handeln. In einer Studie mit angehenden Lehrerinnen und Lehrern ${ }^{2}$ wurden drei mediale Habitustypen herausgearbeitet, von denen jeweils eine Unterform beinhaltet:

- Ambivalente Bürgerliche/mit der Unterform der überforderten Bürgerlichen: Der ambivalent-bürgerliche mediale Habitus bildet sich aus Resten und Versatzstücken eines bildungsbürgerlich-kulturkritischen Habitus. Das «gute Buch» wird hoch angesehen, wohingegen digitale Medien kritisch betrachtet, wenn nicht sogar abgelehnt, werden. Die Medienkompetenz der Gruppe ist eher gering. Die Inhaber dieses Habitus kommen aus einem Elternhaus mit einem hohen kulturellen Kapital.

- Hedonistische Pragmatiker: Die Inhaber des Habitus der hedonistischen Pragmatiker stammen aus einem weniger bildungsbürgerlichen Elternhaus mit einem geringeren kulturellen Kapital. Oft ist das Studium mit einem Bildungsaufstieg verbunden. Die Medien spielen vor allem im Bereich der Unterhaltung eine grosse Rolle und werden wenig hinterfragt. Eine kritische Reflexion spielt keine Rolle.

- Kompetente Medienaffine: Die Inhaber des kompetent-medienaffinen Habitus kommen eher aus einem Haushalt mit überdurchschnittlichem, kulturellem Kapital. Im Elternhaus wurde Wert auf einen selbstverantwortlichen Umgang mit Medien gelegt, wobei diese sorgsam ausgewählt werden und ein Hochkulturschema gepflegt wird. Die Medienkompetenz der Inhaber ist auf das gesamte Medienensemble bezogen, kritisch reflektiert und auch auf einen produzierenden Umgang ausgelegt (vgl. Kommer und Biermann 2012).

2 Wenn von Lehrerinnen und Lehrern gesprochen wird, sind im Folgenden Lehrende aus dem schulischen Kontext gemeint, während sich Lehrende im Artikel auf Lehrende in der Erwachsenenbildung beziehen. 


\section{Der mediale Habitus als Grenze von Medienhandeln}

Die Studie von Kommer und Biermann (Kommer, 2010; Biermann, 2009; Kommer und Biermann 2012; Biermann 2013) zeigt, dass ein Zusammenhang von medialem Habitus und Medienhandeln von (angehenden) Lehrerinnen und Lehrern sowie des medialen Habitus und der Medienkompetenz von Schülerinnen und Schülern vorzufinden ist. Der mediale Habitus bildet hier, wie der Gesamthabitus als Gesamtsystem, ein Untersystem von medienbezogenen Grenzen, das die Vorlieben und Möglichkeiten des Medienhandelns zum einen innerhalb dieser Grenzen beschränkt aber auch ermöglicht. Andere Studien greifen das Analysekonzept des medialen Habitus auf und untersuchen ebenfalls medienpädagogische Fragestellungen damit. So hat eine Studie zu bildungsbezogenem, medialem Habitus untersucht, ob es einen Zusammenhang zwischen medialem Habitus und einer medienbezogenen Wissenskluft bei Grundschulkindern gibt. Die Ergebnisse zeigen, dass schon in diesen frühen Jahren der mediale Habitus der Kinder Dispositionen aufweist, die den Zugang zu Medien und auch zum Medienhandeln fördern oder beschränken (vgl. Henrichwark 2009). Der Einfluss der Primärsozialisation durch die Familie ist gross, so dass die Schule als kompositorische Sozialisationsinstanz diese Unterschiede im Bereich der Medienkompetenzförderung aufgreifen müsste (vgl. ebd.). Der mediale Habitus stellt also schon an dieser frühen Stelle im Bildungsverlauf eine Grenze der Entwicklung der Medienkompetenz dar.

Um entsprechend Medienkompetenz im frühen Bildungsverlauf zu fördern, kommt den Lehrerinnen und Lehrern die wichtige Aufgabe zu, die verschiedenen Voraussetzungen der Kinder zu erkennen und entsprechend medienpädagogisch und medienerzieherisch tätig zu werden. Auch Mutsch (2012) konnte wie Kommer (2010) herausarbeiten, dass es auch im Bereich des medialen Habitus die Gefahr des «clash of habitus» gibt. Sie untersuchte an Wiener Volksschulen die medialen Habitus von Schülerinnen und Schülern und Lehrerinnen und Lehrern und betrachtete die Passung dieser. Es kann davon ausgegangen werden, dass sowohl Schülerinnen und Schüler und Lehrerinnen und Lehrer aufeinander treffen deren medialen Habitus zusammenpassen, als auch eben welche, bei denen diese nicht zueinander passen (vgl. ebd.). Wenn medienpädagogische oder medienerzieherische Prozesse im Unterricht geplant und umgesetzt werden, haben die Schülerinnen und Schüler deren medialer Habitus zu dem der Lehrerin oder des Lehrers passt, einen Vorteil, was, wie in anderen Bildungsbereichen auch, soziale Ungleichheit und Reproduktion dieser unterstützt. Auch an anderer Stelle wurde der Zusammenhang von medialem Habitus der Lehrerinnen und Lehhrer und des schulischen Medienhandelns sowie der Passung der medialen Habitus der Lehrerinnen und Lehrer und Schülerinnen und Schüler bestätigt (vgl. Grubesic 2013). 
Im Projekt Mediengarten wurde das Konzept des medialen Habitus bei einer Untersuchung von 3- bis 6-Jährigen als Analysekategorie verwendet. In dem Projekt wurde die Medienkultur der Kinder und in diesem Zusammenhang der frühe mediale Habitus untersucht. Die Ergebnisse lassen vermuten, dass gerade in dem jungen Alter das kulturelle Kapital der Familie eine besondere Rolle im Bereich des medialen Habitus spielt, da selbst bei ähnlicher Medienausstattung sich Unterschiede in Mediennutzungstechniken zeigten, die sich an den Leitmedien in der Kernfamilie orientieren (vgl. Swertz, Kern, und Kovacova 2014).

Betrachtet man die Professionalisierungsvoraussetzungen der Lehrenden in der Erwachsenenbildung und die von Lehrerinnen und Lehrern, dann kann im schulischen Kontext eher davon ausgegangen werden, dass die Lehrerinnen und Lehrer im Verlauf ihrer strukturellen Professionalisierung, z.B. während des Studiums oder bei medienpädagogischen Fortbildungen, mit medienpädagogischen Themen konfrontiert werden und die Lehrerinnen und Lehrer die Inhalte dann je nach ihren habituellen Voraussetzungen einordnen - auch wenn es keine einheitliche Verankerung von medienpädagogischen Inhalten in lehrerbildenden Studiengängen gibt. Wie die oben genannten Studien gezeigt haben, stellt der mediale Habitus hier Grenzen dar, die zum einen Medienhandeln einschränken, zum anderen aber auch ermöglichen. Auch für den medialen Habitus - als Teil des Gesamthabitus - gilt, dass er nicht unveränderlich ist. Im Bereich der Lehrerbildung besteht hier eine grössere Wahrscheinlichkeit zumindest Berührungspunkte mit medienpädagogischen Inhalten geboten zu bekommen, die zu Irritation führen könnten, so dass die Möglichkeit der Reflexion von Einstellungen des eigenem Medienhandeln angestossen werden kann. Bei den Lehrenden in der Erwachsenenbildung fallen diese strukturellen Berührungspunkte zum grossen Teil weg. Die Lehrenden gestalten ihre Professionalisierungswege in der Regel autark und entscheiden selbst, mit welchen Inhalten sie sich formal, nonformal oder auch informell auseinandersetzen. Lehrende mit einem den digitalen Medien eher negativ eingestellten Habitus werden dementsprechend eher vermeiden, sich in diesem Bereich fortzubilden und zu professionalisieren als medienaffine Lehrenden. Hierbei spielt der Aspekt der Hysteresis (d.h. der Trägheit des Habitus) eine Rolle, also die Tendenz des Habitus in dem zu verharren, was man mag und kennt (vgl. Lenger, Schneickert, und Schuhmacher 2013).

Für den Bereich der Lehrerbildung kommt Kommer (2010) zu dem Ergebnis, dass der Habitus zum einen entscheidend dafür ist, wie Medien und Medieninhalte eingeordnet werden, zum anderen aber auch Grenzen darstellt, welche Medien wahrgenommen werden. Das medienbezogene Lehrhandeln der «verunsicherten, bürgerlichen» angehenden Lehrerinnen und Lehrer wird von daher eher nicht von mediendidaktischen und medienpädagogischen Einsatz digitaler Medien geprägt sein, da diese keinen Teil ihres medialen Handlungsspielraums darstellen (vgl. ebd.). 
Wenn die Lehrenden in der Erwachsenenbildung in ihrem Handeln durch ihre habituellen Präferenzen beeinflusst werden, muss davon ausgegangen werden, dass der mediale Habitus als System von Grenzen einen noch grösseren Einfluss auf das berufliche Medienhandeln hat, da für medienpädagogische Professionalisierung wenig äussere Anreize vorhanden sind. Schon in frühen Bildungsphasen, wenn der mediale Habitus noch kein festes Dispositionssystem wie im Erwachsenenalter darstellt, erweist sich der mediale Habitus als habituelles Untersystem als Grenze für die Entwicklung von medienbezogenen Handeln und Medienkompetenz. Es kann davon ausgegangen werden, dass ohne Anreize zur Reflektion und Irritation des medialen Habitus' aufgrund der primären individuellen Professionalisierung der Lehrenden in der Erwachsenenbildung, der mediale Habitus einen besonders grossen Einfluss auf die Entscheidungen hat, in welchen Bereichen medienpädagogische Professionalisierung stattfindet. Betrachtet man das Modell der medienpädagogischen Kompetenzanforderungen an Lehrende in der Erwachsenenbildung, kann der mediale Habitus auf alle medienpädagogischen Kompetenzfacetten durch entsprechende Professionalisierungsentscheidungen wirken. Die aufgeführten Studien zeigen, dass der mediale Habitus Auswirkungen auf die allgemeine Medienkompetenz, auf die mediendidaktische Umsetzung und auf die Einstellungen und Werthaltungen gegenüber Medien im schulischen Lehr-/Lernkontext hat. In der Erwachsenenbildung gibt es bisher noch keine Untersuchungen der Auswirkungen des medialen Habitus auf den Lehr-/Lernkontext. Um medienpädagogische Professionalisierungsangebote für Lehrende in der Erwachsenenbildung zu entwickeln - die möglichst viele Lehrende mit unterschiedlichen medialen Habitustypen erreichen - stellt der mediale Habitus eine Analysekategorie dar, die die Grenzen der medienpädagogischen Professionalisierung aufdecken kann, die somit in der Gestaltung der Angebote berücksichtig und reflektiert werden können.

\section{Literatur}

Biermann, Ralf. 2009. Der mediale Habitus von Lehramtsstudierenden - Eine quantitative Studie zum Medienhandeln angehender Lehrpersonen. Wiesbaden: VS Verlag für Sozialwissenschaften.

Biermann, Ralf. 2013. «Medienkompetenz - Medienbildung - Medialer Habitus. Genese und Transformation des medialen Habitus vor dem Hintergrund von Medienkompetenz und Medienbildung». Medienimpulse - Beiträge zur Medienpädagogik, Nr. 4. http://www.medienimpulse.at/articles/view/604

Bolten, Ricarda, und Karin Julia Rott. 2018. «Medienpädagogische Kompetenz: Anforderungen an Lehrende in der Erwachsenenbildung - Perspektiven der Praxis» MedienPädagogik: Zeitschrift für Theorie und Praxis der Medienbildung 30 (März): 137-53. https://doi. org/10.21240/mpaed/30/2018.03.05.X. 
Bourdieu, Pierre. 1987. Die feinen Unterschiede. Kritik der gesellschaftlichen Urteilskraft. Frankfurt/Main: Suhrkamp.

Bourdieu, Pierre, und Loïc J. D. Wacquant. 2006. Reflexive Anthropologie. Frankfurt/Main: Suhrkamp.

European Commission. 2015. «Education and Training 2020. Improving Policy and Provision for Adult Learning in Europe». http://ec.europa.eu/education/library/reports/policy-provision-adult-learning_en.pdf.

Faulstich, Peter. 2009. "Lernorte - Flucht aus der Anstalt» In Lernorte. Vielfalt von Weiterbildungs- und Lernmöglichkeiten, herausgegeben von Peter Faulstich und Mechthild Bayer, 7-27. Hamburg: VSA-Verlag.

Gapski, Harald, Monika Oberle, und Walter Staufer. 2017. «Einleitung»In Medienkompetenz. Herausforderung für Politik, politische Bildung und Medienbildung, herausgegeben von Harald Gapski, Monika Oberle und Walter Staufer, 17-30. Bonn: Bundeszentrale für politische Bildung.

Grubesic, Katharina. 2013. «Medienbildung in Der Volksschule. Eine Empirische Untersuchung Zum Medialen Habitus von LehrerInnen». medienimpulse - Beiträge zur Medienpädagogik, Nr. 4. http://www.medienimpulse.at/articles/view/601.

Henrichwark, Claudia. 2009. «Der Bildungsbezogene Mediale Habitus von Grundschulkindern - Eine Empirische Studie zur Reproduktion sozialer Ungleichheit in Schule Und Familie». Dissertation, Bergische Universität Wuppertal. http://nbn-resolving.de/ urn:nbn:de:hbz:468-20090741.

Koller, Hans-Christoph. 2012. Bildung anders denken. Einführung in die Theorie transformatorischer Bildungsprozesse. Stuttgart: Kohlhammer.

Kommer, Sven. 2010. Kompetenter Medienumgang? Eine qualitative Untersuchung zum medialen Habitus und zur Medienkompetenz von SchülerInnen und Lehramtsstudierenden. Opladen und Farmington Hills MI: Budrich UniPress.

Kommer, Sven, und Ralf Biermann. 2012. "Der mediale Habitus von (angehenden) Lehrerlnnen. Medienbezogene Dispositionen und Medienhandeln von Lehramtsstudierenden». In Jahrbuch Medienpädagogik 9: Qualitätsentwicklung in der Schule und medienpädagogische Professionalisierung, herausgegeben von Renate Schulz-Zander, Birgit Eickelmann, Heinz Moser und Horst Niesyto, 81-108. Wiesbaden: VS Verlag für Sozialwissenschaften. https:// doi.org/10.1007/978-3-531-94219-3.

Lenger, Alexander, Christian Schneickert, und Florian Schumacher. 2013. «Pierre Bourdieus Konzeption des Habitus». In Pierre Bourdieus Konzeption des Habitus. Grundlagen, Zugänge Und Forschungsperspektiven, herausgegeben von Florian Lenger, Alexander Schneickert und Christian Schumacher, 13-41. Wiesbaden: Springer. https://doi.org/10.1007/978-3531-18669-6.

Martin, Andreas, Stefanie Lencer, Josef Schrader, Stefan Koscheck, Hana Ohly, Rolf Dobischat, Arne Elias, und Anna Rosendahl. 2017. Das Personal in Der Weiterbildung. Bielefeld: W. Bertelsmann. https://doi.org/103278/85/0015w. 
Michel, Burkard. 2010. «Habitus und Lebensstil»In Handbuch Mediensozialisation, herausgegeben von Ralf Vollbrecht und Claudia Wegener, 75-84. Wiesbaden: Springer VS.

Mutsch, Ursula. 2012. "Der Mediale Habitus von Volksschulkindern Und Ihren Lehrerinnen und Lehrern». Dissertation, Universität Wien. http://othes.univie.ac. at/23971/1/2012-10-18_0104284.pdf.

Otto, Volker. 1979. Offenes Weiterlernen. Erwachsenenbildung im Selbstlernzentrum. Braunschweig: Westermann.

Podehl, Bernd. 1984. Medienpädagogik und Erwachsenenbildung. Frankfurt am Main: Fischer.

Rohs, Matthias, und Ricarda Bolten. 2017. «Medienpädagogische Professionalisierung von Erwachsenenbildner*Innen. Eine Biographische Annäherung» Beiträge Zur Erwachsenenbildung, Nr. 4. https://kluedo.ub.uni-kl.de/files/4547/_Beitraege_zur_EB_4_Medienkompetenz.pdf.

Rohs, Matthias, Ricarda Bolten, und Jonathan Kohl. 2017. «Medienpädagogische Kompetenzen in Kompetenzbeschreibungen für Erwachsenenbildner*Innen» Beiträge zur Erwachsenenbildung 5. https://kluedo.ub.uni-kl.de/files/4690/_Beitraege_zur_EB_5_final.pdf.

Rosenberg, Florian von. 2011. Bildung und Habitustransformation. Empirische Rekonstruk- tionen und bildungstheoretische Reflexionen. Bielefeld: transcript.

Schmidt-Hertha, Bernhard, Matthias Rohs, Karin Julia Rott, und Ricarda Bolten. 2017. «Fit Für Die Digitale (Lern-)Welt?» DIE Zeitschrift Für Erwachsenenbildung 3: 35-37. https://doi. org/10.3278/DIE1703W.

Stang, Richard. 1996. «Wahrnehmungsbildung als Zukunftsaufgabe» In Medienkompetenz als Schlüsselbegriff, herausgegeben von Antje von Rhein, 141-155. Stuttgart: Klinkhardt.

Swertz, Christian, Gudrun Kern, und Erika Kovacova. 2014. «Der Mediale Habitus in der Frühen Kindheit» MedienPädagogik: Die Zeitschrift für Theorie und Praxis der Medienbildung 22 (Dezember): 1-28. https://doi.org/10.21240/mpaed/22/2014.12.15.X. 